\title{
Roles of Seed Components in the Growth of Barley Seedlings under Salt Stress
}

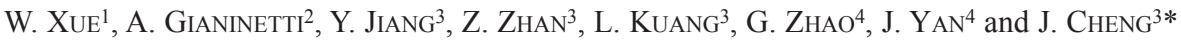 \\ ${ }^{1}$ College of Life Sciences, Guizhou University, Guiyang, Guizhou 550025, China \\ ${ }^{2}$ Consiglio per la ricerca in agricoltura e l'analisi dell'economia agraria, Research Centre for Genomics and \\ Bioinformatics, Fiorenzuola d'Arda, PC 29017, Italy \\ ${ }^{3}$ College of Agriculture, Guizhou University, Guiyang, Guizhou 550025, China \\ ${ }^{4}$ School of Pharmacy and Bioengineering, Chengdu University, Chengdu, Sichuan 610106, China
}

(Received 28 July 2017; Accepted 11 January 2018;

Communicated by X.F. Zhang)

The cereal endosperm provides nutrients for seedling growth. The effects of seed components in seedling establishments under salt stress are, however, not yet fully explored. In this study, 60 barley recombinant inbred lines derived from Lewis $\times$ Karl cross were grown in four different environments, and the seed contents of starch, total soluble protein, phytate, total phenolics, total flavonoids and total inorganic phosphorus were determined in the harvested grains. Seeds of each line from the four environments were also assayed for seedling growth under saline treatments from 0 to $400 \mathrm{mM} \mathrm{NaCl}$. Root and shoot lengths after 7 days decreased with increasing salt concentration. Correlations between seed components and either root or shoot length were established across the four seed sources. ANOVA showed a significant environment/source effect for both seed components and seedling growth, although the latter was less affected by the seed-production environment. Modeling seedling length across multiple salinities for each seed source showed that the environment with the most saline-tolerant root-growth curve was that associated the highest seed phosphorus content. Correlations between seed components and seedling growth traits highlighted phytate and total inorganic phosphorus as key components for seedling growth under moderate salinities. Seed phytate contents benefited seedling growth, even at high salinities, suggesting an additional role for this seed component under stressful growth conditions, possibly linked to its potential function as an osmolyte source.

Keywords: seed component, salt tolerance, seedling, multiple seed source, phytate

\section{Introduction}

Seed germination is the morphological and physiological process by which mature seeds are transformed into seedlings. Chemically, the many components that fill the grains during seed development are further mobilized for seedling establishment. A few studies have elucidated the filling rate of specific seed components in developing seeds, and their effect on further germinating capacity, for example, a study with barley starch-accumulat-

\footnotetext{
*Corresponding author; E-mail: jpcheng@gzu.edu.cn; Phone: +86 13765006312; Fax: +86 8513855894
} 
ing mutants that are susceptible to preharvest sprouting (Howard et al. 2012). Potential roles of seed components in furthering germinating behavior have been proposed and tested in vegetable seeds, showing positive correlations between seed fatty acid content and germination rate (Kaymak 2012), and similar patterns of soybean seed phytate and oligosaccharide contents with field emergence (Meis et al. 2003). However, the role of barley seed components in seedling growth under abiotic stress is still unknown.

Soil salinization is a serious threat to field crops, which employs two distinct mechanisms to deal with the ionomic and osmotic effects of salinity, each on a different timescale (Zhu et al. 2015). Even though the ionic effects cannot be underestimated, osmotic responses and adjustments are crucial for crop survival under moderate salt stress, for instance, proline and glycine accumulation in crop roots and young leaves (Hajlaoui et al. 2010). The osmotic effect is also considered the major outcome of exposure to salt during seed germination (Zhang et al. 2010), suggesting putative roles for seed osmolytes in maintaining low osmotic potential for water uptake during imbibition.

Seeds are composed of multiple components that serve not only as energy sources, but possibly also as osmolytes for seedling emergence under salt stress, such as albumins and globulins which are soluble in salt solutions (Witzel et al. 2010). Carbon (C) and nitrogen (N) are major elements in barley seeds, and phosphorus (P) is also essential for rapid seedling establishment (White and Veneklaas 2012), mainly stored as organic phytate (Phy) (Raboy 2009). Micro-amounts of antioxidants in seeds, such as phenolics (Phe) and flavonoids (Fla), also play an important role in seed germination (Zhou et al. 2010).

Although seed macro- and micronutrients are largely affected by environment (Xue et al. 2016), there have been few relevant studies on the effect of this on seed germination and seedling traits. Instead, major studies have been dedicated to investigating the environmental effects on seed germination, such as, the revelation of 561 environmental factor-responsive proteins from cereal and vegetable seedlings under different experimental conditions (Tan et al. 2013). Our study was to determine how seedling growth is affected by diverse seed sources (environments). We therefore examined whether seeds derived from different environments exhibit equivalent variations in seedling growth and responses to salt stress, and estimated the correlations between seed components and stressed-seedling traits using evidence from multiple environmental sources for seed production.

\section{Materials and Methods}

\section{Field trials and seed component measurements}

Sixty lines derived from a Lewis $\times$ Karl (LK) recombinant inbred line (RIL) population plus the two parents were propagated under four field conditions, using three replicates in a complete randomized block design. Two conditional environments during the 2012 2013 season (Y2013-Fertilized and Y2013-Non-Fertilized, abbreviated to Y2013-F and Y2013-NF) were established at Site $1\left(26^{\circ} 15^{\prime} \mathrm{N}, 105^{\circ} 55^{\prime} \mathrm{E}\right)$ in P.R. China as previously described (Xue et al. 2016), and an additional two experiments were conducted at Site 2 
$\left(3212^{\prime \circ} \mathrm{N}, 118^{\circ} r 9^{\prime} \mathrm{E}\right)$ and Site $3\left(32^{\circ} 12^{\prime} \mathrm{N}, 118^{\circ} 30^{\prime} \mathrm{E}\right)$ in Anhui province of P.R. China during the 2013-2014 season (Y2014A1 and Y2014A2). Analyses of soil samples from the three sites, as well as of seed contents of starch (Sta), total soluble protein (TSP), Phy, Phe, Fla and inorganic $\mathrm{P}\left(\mathrm{P}_{\mathrm{i}}\right)$ were performed as previously described (Xue et al. 2016).

\section{Phenotyping seedling traits under different salinities}

A vertical seedling growth system, which has been described by Xue et al. (2017), was used to germinate the collected seeds in 80,160, 240, 320 and $400 \mathrm{mM}$ sodium chloride $(\mathrm{NaCl})$ solution, in parallel with control condition (no salt added). All "seeds" (referring in the wide sense to the barley grain spikelet) were incubated for 7 days in a growth chamber at $20{ }^{\circ} \mathrm{C}$ without light. The resultant seedlings were photographed and graphically measured by Image $\mathrm{J}$ for root and shoot length (RL and SL, respectively), or coleoptile length in some treatments (regarded as SL). The obtained data were subjected to simulation with the Hill-3-parameter mathematical model

$$
\left(y=\frac{a x^{b}}{c^{b}+x^{b}}\right)
$$

(Joosen et al. 2010) to draw dynamic curves of seedling length ( $y$ axis) against salinity ( $x$ axis) by Sigmaplot 12.0 (Systat Software, Inc., 2012, USA), wherein $a$ is a limit value for the maximum seedling length $\left(L_{\max }\right), b$ controls the shape and steepness of the simulated curve, and $c$ is the half-maximal activation level of the curve (Xue et al. 2017). Coleoptiles rarely emerged in the $400 \mathrm{mM} \mathrm{NaCl}$ treatment, and seedlings without coleoptiles were recorded as having an SL value of 0 for further data modeling.

\section{Statistics}

Two-way ANOVA was carried out by STATISTICA7 (StatSoft, Inc., 2004, USA), considering genotype $(\mathrm{G})$ as a fixed factor and environment $(\mathrm{E})$ as a random one. Tukey HSD was applied with JMP 6.0 software (SAS Institute, Inc., 2005, USA). Principal component analysis (PCA) was calculated by free software PAST (http://folk.uio.no/ohammer/ past/) and illustrated by Sigmaplot 12.0. Pearson correlation matrix was calculated by R 2.1.1 (https://www.r-project.org/) using the data from the four environments, and significant correlations $(p<0.05)$ were visualized as networks by Cytoscape 2.8.1 (http://www. cytoscape.org/). Trait SL-400 mM (shoot length under $400 \mathrm{mM} \mathrm{NaCl}$ ) was not included in this section of the statistical analysis because only $39 \%$ of the lines showed some (a very few) seedlings with an emerged coleoptile across all the 4 environments. 


\section{Results}

Seed-source effects on seed components and seedling traits under different levels of salinity

Sixty barley lines and two parents were previously propagated in two conditional environments at Site 1 (Y2013F and Y2013NF), and expanded to another two fields (Sites 2 and 3) in season 2013-2014 (Y2014A1 and Y2014A2, respectively). The soil conditions at the three sites are presented in Table $\mathrm{S} 1^{*}$. Significant variation in soil available $\mathrm{P}$ $\left(\mathrm{P}_{\text {available }}\right)$ content was observed among the three sites, being highest at Site 2 and lowest at Site 3. This resulted in significantly higher seed Phy and $\mathrm{P}_{\mathrm{i}}$ contents in Y2014A1 compared to the other environments (Fig. S1). However, seed contents of the other micronutrients, such as seed Phe and Fla, did not follow this pattern. High TSP contents were found in both Y2014A1 and Y2014A2, but notably lower Sta contents were found in Y2014A2 relative to the other sites, suggesting Y2014A1 (Site 2) as the most beneficial environment for seed nutrition.

To evaluate environments that differed from the others for either seed components or seedling growth, PCA was employed. Y2014A1 was isolated from the other three environments along PC1 in the PCA results for seed components (Fig. 1A). PC1 explained $67.8 \%$ of the total variation, with a main contribution from the variables Phy and Pi contents, consistent with the earlier results. No clear separation was observed along PC2 among the environments, where variations in Fla dominated. Notably, minor variations in seedling traits are displayed in Fig. 1B among the four environments. To estimate the seed-source effect on seed components, seedlings non-stressed and salt-stressed seed-

Seed Components

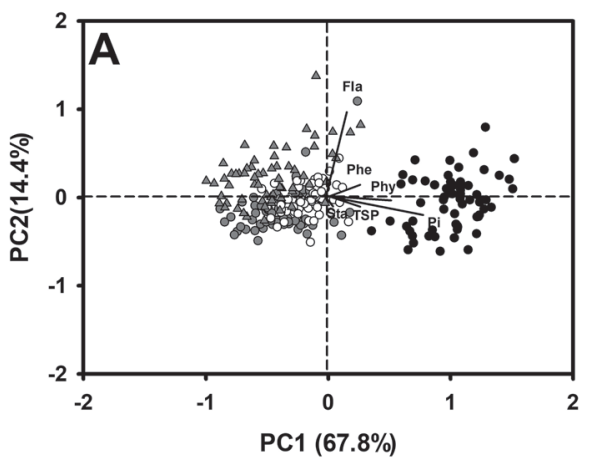

\section{Seedling Traits}

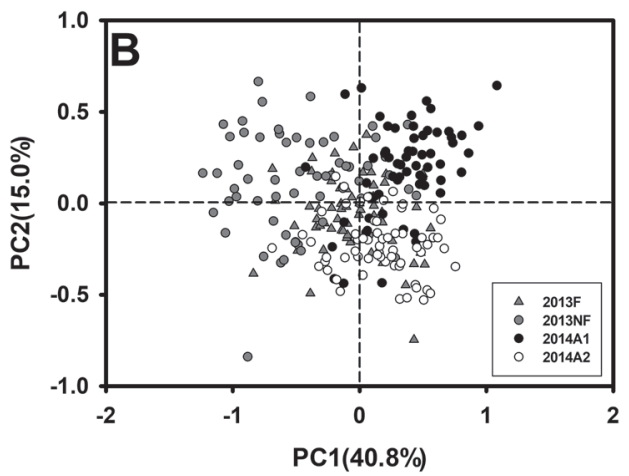

Figure 1. Principal component analysis of studied traits, barley seed components (A), and seedling growth under normal and salt-stress conditions (B). The explained variations of each component are labeled within the graphs. Different environments are distinguished by symbols. The loading plot for the two principal components is also presented in graph A

*Further details about the Electronic Supplementary Material (ESM) can be found at the end of the article. 
lings, two-way ANOVA was conducted (Tables S2 and S3). A large environmental contribution (52-65\%) was noted for all seed components except for the small contribution for Fla $(17 \%)$. This contribution decreased to an average of $14 \%$ in non-stressed and stressed seedling traits, with only $4 \%$ environmental contribution observed for traits SL-0 mM and SL-80 mM, respectively. These results indicated that the seedling traits are significantly affected by seed source, but less influenced compared to the environmental effects on the seed components. Seed Fla content made the highest genotype contribution among all seed components, in contrast to Sta and Phy which were not significant genotype factors. Comparatively, contributions of genotype factors were clearly enhanced in seedling traits, wherein RL-240 mM gave the highest score. Similarly, strong genotype $\times$ environment interactions (GEIs) were also achieved for most of seedling traits, but not for all the seed components.

\section{Dynamic responses of seedlings to salt stress and correlations with seed components}

A sigmoidal model (Hill function) was used to simulate seedling length under the different salinities, and dynamic curves for seedling responses to salt stress are depicted in Fig. 2. Clear variations were predicted among RL rather than SL curves. Similar to the PCA results

in

Fig. 1B, there was nearly no separation on the RL curves between Y2013F and Y2013NF, indicating that fertilization status does not differ for seedling roots under salt stress. However, a tolerant root response to salinity was simulated in Y2014A1 relative to Y2014A2 (Fig. 2), which predicted that P content in seeds from Y2014A1 (PCA results in Fig. 1A) benefits root growth under salinity.

Pearson correlations between seed components and seedling traits are presented in Fig. S2. Sta content was positively correlated to seedling traits in treatments with no or low salinity, but this correlation became negative at high salinities. TSP gave only posi-

RL

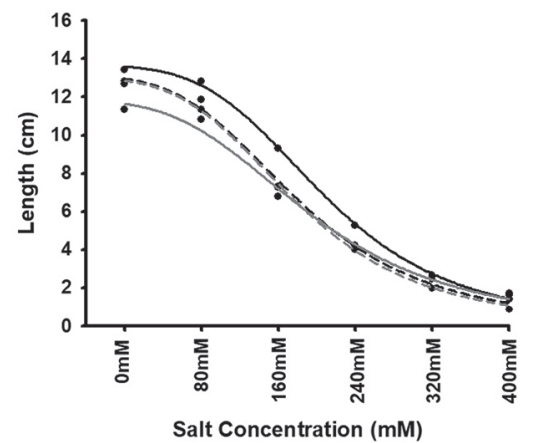

SL

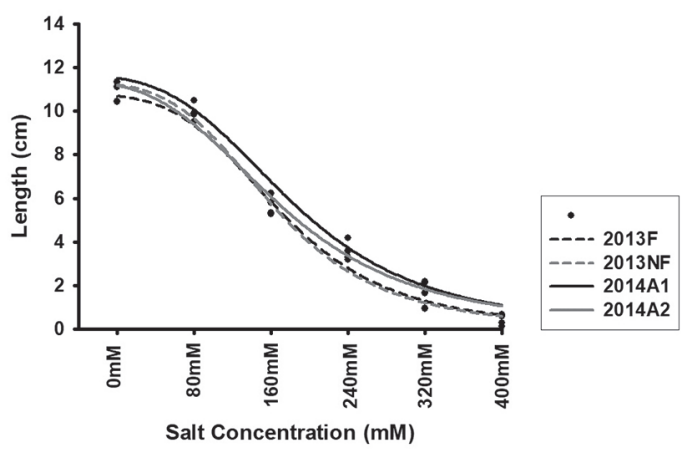

Figure 2. Dynamic responses of root length (RL) and shoot length (SL) under different salinities. Each point represents the average RL and SL for a single environment, after 7 days of germination and seedling growth under different salt-stress conditions. Different environments are distinguished by colors. All of the curve-fitting coefficients are higher than 0.99 
tive correlations with seedling traits. Phe displayed a few positive correlations, in contrast to Fla which showed no significant links with seedling traits from either control or salinity treatments. This indicated useful roles for seed Phe, but not Fla contents in seedlings. Both Phy and $\mathrm{P}_{\mathrm{i}}$ showed strong positive correlations with multiple seedling traits (described in next section).

\section{The relationships between seed P source and conditional seedling growth}

To discuss the notable roles of Phy and $\mathrm{P}_{\mathrm{i}}$ in the correlation-based networks, a detailed characterization of the association between seed $\mathrm{P}$ and seedling traits is presented in Fig. 3, where the coefficients are labeled for each significant link. Phy was positively correlated with both $R L$ and $S L$ traits, but $P_{i}$ was only significantly related to RL traits. The correlations of $\mathrm{P}_{\mathrm{i}}$ content to root growth were relatively elevated with increasing salinity ( $r$ from 0.327 to 0.427 ), and the strongest correlation was observed between $\mathrm{P}_{\mathrm{i}}$ and RL$160 \mathrm{mM}(r=0.551)$. No significant relations were found at the treatment with the salt concentration above $240 \mathrm{mM} \mathrm{NaCl}$.

Similar trends were also displayed by Phy, with strong correlations between Phy and RL ( $r$ from 0.387 to 0.438 ) that gradually decreased to the $240 \mathrm{mM} \mathrm{NaCl}$ treatment

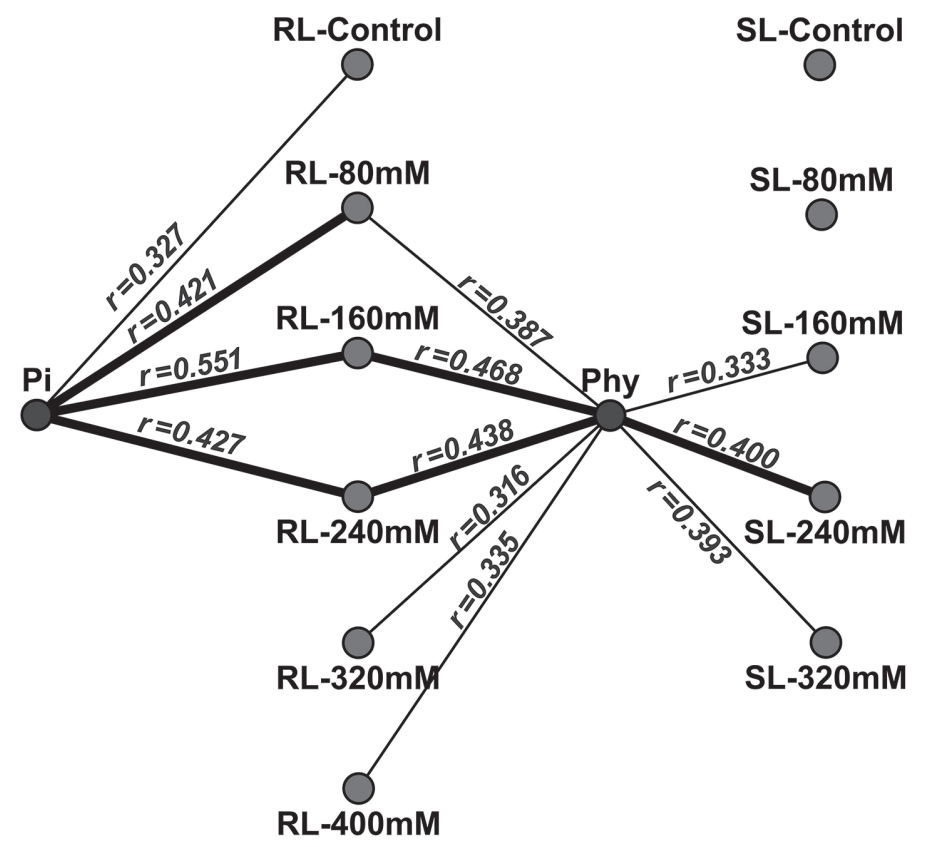

Figure 3. Correlations between seed $\mathrm{P}$ content and seedling traits. The correlations are conducted with the dataset from four environments. The solid lines refer to the positive Pearson correlations. Wider lines indicate $r>0.4$, and all significant correlations are labeled with their corresponding coefficients 
( $r$ from 0.438 to 0.335 ). Notably, Phy was significant linked with all RL traits except RLControl, and all stressed SL traits except SL- $80 \mathrm{mM}$. Referring to the individual treatments, seed $\mathrm{P}_{\mathrm{i}}$ content initially exhibited a high correlation with non-stressed root growth, and in parallel with the Phy correlations, gradually starting from the low-salt-stress treatment $(80 \mathrm{mM} \mathrm{NaCl})$. This characteristic was clearly seen with the 160 and $240 \mathrm{mM} \mathrm{NaCl}$ treatments, where seed P sources were able to link all RL and SL traits. However, only Phy showed correlations with seedling traits at high salinities (320 and $400 \mathrm{mM}$ ), implying additional functions of Phy under these circumstances.

\section{Discussion}

The effects of seed source on seedling growth are partially dependent on its effects on seed components

The dominant environmental effects and large contributions of GEI on barley seed components have been discussed (Xue et al. 2016). Similar results were obtained in this study for macronutrients (Sta and TSP), which exhibited a smaller GEI than the micronutrients. However, seedling growth still significantly varied between seeds from different environmental sources. In maize, high heritability of seedling traits was observed in two experimental fields, and thus many QTLs could be identified (Hund et al. 2004). In rice, a major seedling QTL from a single environment contributed over $40 \%$ phenotypic variation (Andaya and Mackill 2003). However, germination tests on Brassica seeds collected from two different seed sources confirmed a strong environmental effect, whereas a genome-wide association study suggested that germination could nevertheless be substantially improved by genome-based breeding, demonstrating an important role for genotype as well (Hatzig et al. 2015).

In our study, significant effects of both environmental seed source and genotype factor were observed on traits such as SL-Control and RL-240 mM (Table S3). The significant role played by the genotype factor in seedling traits depended on salinity treatment, and differed between shoot and root. Shoot growth was strongly genotype-dependent under non-stressed conditions, but this genetic control gradually weakened (from $21 \%$ to $13 \%$ ) with increasing salt concentration (Table S3). In contrast, there was no significant contribution of genotype in RL-Control, but it increased gradually (from $11 \%$ to $22 \%$ ) with increasing salinity up to $240 \mathrm{mM} \mathrm{NaCl}$ (Table S3). This indicated a strong genetic effect on root growth under moderate salt stress, as had already been demonstrated by the discovery of major QTLs in the OWB barley population (Witzel et al. 2010) and NT barley population (Xue et al. 2017). Hence, our results suggest that potential genetic influence on barley seedling traits is organ- (shoot vs. root) and condition-dependent. Even though the environmental seed-source effects had much less impact on seedlings relative to seed components, seeds with a more favorable composition produced the best dynamic curve of root response to salinity (Fig. 2). This implies additive benefits from seed nutrition for seedling growth. 


\section{Potential roles of Sta, TSP and Phe in seedling growth under salt stress}

In this study, Sta, TSP and Phe components exhibited valuable correlations with nonstressed seedling growth, and even with growth under salt stress (Fig. S2). Sta is the main $\mathrm{C}$ source for seedling establishment, and seeds with higher Sta content can make full use of this storage reservoir under low or no salinity stress; however, it becomes largely nonutilized when growth is strongly restricted, with the high Sta content actually diluting those of the other seed components which might have favorable functions under high salinity.

Storage proteins in barley seeds include albumins, globulins and hordeins. Large mobilization of storage glutelins was observed in response to salt stress in rice (Xu et al. 2016) and cashew seeds (Voigt et al. 2009). Those results suggested a positive role for storage proteins in seedling growth under salt stress, as confirmed here by the observed positive correlation between TSP and seedling growth at high salinities. In fact, some amino acids derived from protein degradation can act as osmolytes and increase the seedling's resistance to salt stress (Mansour 2000). Phe play positive roles in several antioxidant activities, which might explain the positive effect of Phe on seedling growth at high salinity, when ROS production can be induced by the strong stress (Piwowarczyk et al. 2016).

\section{Seed $P$ and Phy contents are environmentally determined and can have non-entirely overlapping roles in seedling growth}

A large number of studies have focused on $\mathrm{P}$ uptake and translocation by roots, but only a few have addressed seed P content (Raboy 2009). The main effector of P uptake is soil available P content (Wang et al. 2016), which is also in agreement with our results for both Phy and $\mathrm{P}_{\mathrm{i}}$ contents in seeds. Correspondingly, ANOVA of seed Phy among different environments suggested a non-significant genotypic effect (Table S2), which is consistent with the non-polymorphic sequencing results observed for the key Phy-synthesis gene HvMIPS1 between the two parents (data not shown). Hence, it suggested that the major variations in seed $\mathrm{P}$ in our study were due to different field conditions.

In accordance with the present findings, exogenous $P$ supply can increase the salt tolerance of barley seedlings (Al-Karaki 1997). Moreover, a study on the interaction between P supply and salt stress emphasized the dominant role of P availability (Talbi Zribi et al. 2011), although endogenous P provided only a small contribution to seedling salt tolerance. Notably, in our experiment, barley seeds with elevated seed P content also showed better seedling growth, which is consistent with a previous study in barley (Zhang et al. 1990). In general, a number of studies have reported that seeds obtained from plants grown in P-deficient soils have lower P concentrations than seeds obtained from plants grown in high-P soils, and also show poorer seedling establishment (Rose et al. 2013; White and Veneklaas 2012). Interestingly, however, our correlations between seed P content and seedling traits (Fig. 3) also exhibited dynamics that shifted with the different salinities. Correlations characterized good positive connections between $\mathrm{P}_{\mathrm{i}}$ and root traits 
under no or low salt stress (control and $80 \mathrm{mM}$ ), further complemented by Phy content under moderate salt stress (160 and $240 \mathrm{mM}$ ), but dominated only by Phy content under high salinities (320 and $400 \mathrm{mM}$ ). This finding indicated some differential functions of Phy with respect to total inorganic $P$.

The total net $\mathrm{P}$ content from Phy in our seeds represents an important source of $\mathrm{P}_{\mathrm{i}}$, but stepwise Phy hydrolysis requires various phosphatases and phytases, such as purple acid phosphatase and inositol monophosphate (Suzuki et al. 2007), which might not be able to supply $\mathrm{P}$ rapidly. Instead, free $\mathrm{P}_{\mathrm{i}}$ is an immediately available $\mathrm{P}$ source, which might explain its strong connection with root growth under no, low and moderate salt-stress conditions (from 0-240 $\mathrm{mM} \mathrm{NaCl}$ ). However, Phy started to display a significant role in seedling growth from $160 \mathrm{mM} \mathrm{NaCl}$, especially for shoot growth. This might be attributed to the increased osmotic effects of elevated salinity, because in barley, the major stress component of high salinity seems to be osmotic (Zhang et al. 2010).

Overexpression of the gene encoding myo-inositol 3-phosphate synthase clearly enhances salt tolerance in Arabidopsis and rice seedlings, mainly via the consequent increase in inositol (Joshi et al. 2013; Raychaudhuri and Majumder 1996). Furthermore, inositol accumulation in seedlings under salt stress has been demonstrated (Klages et al. 1999; Saxena et al. 2013). The significant decrease in Phy content and increase in phytase activity were also observed in lettuce seedling under salt stress (Nasri et al. 2011). Therefore, as the major form of stored $\mathrm{P}$ in the seed, we hypothesized it might also be an ideal inositol source for osmotic adjustment under high salinities. On the one hand, P amount was not a significant factor for seedling growth under high salinities in our experiment, since no significant $\mathrm{P}_{\mathrm{i}}$-related correlations were observed above $240 \mathrm{mM} \mathrm{NaCl}$. On the other, inositols are biologically compatible solutes accumulated by cells in response to salt, even though their role as osmolytes is not clear (Michell 2007). So, as Phy also constitutes a reserve of inositols, it can be hypothesized that these might be crucial for seedling growth under high salinities and therefore, under those conditions, seedling growth is significantly correlated with seed Phy content. Indeed, water stress and salt stress can increase the concentration of the osmolyte myo-inositol in plants (Schneider et al. 2006). Whether the inositol derived from degraded Phy responding to osmotic adjustments under high salinity really acts as an osmolyte still needs to be confirmed, and this will be the topic of future studies.

In conclusion, our results confirmed a larger impact of environmental seed source on seed components than on seedling traits. However, better seed composition under favorable field conditions still benefits its derived seedling with improved rootlet responses to salt stress, especially as regards the significant roles of seed Phy and $\mathrm{P}_{\mathrm{i}}$ content, which are mainly determined by soil available $\mathrm{P}$ content. Correlation analysis showed that higher seed Phy content, rather than high $\mathrm{P}_{\mathrm{i}}$ content, is associated with better seedling growth under high salinities, suggesting its role not only as a P source but also as an osmolyte source. 


\section{Acknowledgements}

This work was supported by program of Natural Science Foundation of China (NO: 31560578), and by a China-Israel cooperation program grant from the Ministry of Science and Technology in China (No. 2013DFA32200).

\section{References}

Al-Karaki, G.N. 1997. Barley response to salt stress at varied levels of phosphorus. J. Plant Nutr. 20:16351643.

Andaya, V.C., Mackill, D.J. 2003. Mapping of QTLs associated with cold tolerance during the vegetative stage in rice. J. Exp. Bot. 54:2579-2585.

Hajlaoui, H., Ayeb, N.E., Garrec, J.P., Denden, M. 2010. Differential effects of salt stress on osmotic adjustment and solutes allocation on the basis of root and leaf tissue senescence of two silage maize (Zea mays L.) varieties. Ind. Crop. Prod. 31:122-130.

Hatzig, S.V., Frisch, M., Breuer, F., Nesi, N., Ducournau, S., Wagner, M.H., Leckband, G., Abbadi, A., Snowdon, R.J. 2015. Genome-wide association mapping unravels the genetic control of seed germination and vigor in Brassica napus. Front. Plant Sci. 6:221.

Howard, T.P., Fahy, B., Craggs, A., Mumford, R., Leigh, F., Howell, P., Greenland, A., Smith, A.M. 2012. Barley mutants with low rates of endosperm starch synthesis have low grain dormancy and high susceptibility to preharvest sprouting. New Phyto. 194:158-167.

Hund, A., Fracheboud, Y., Soldati, A., Frascaroli, E., Salvi, S., Stamp, P. 2004. QTL controlling root and shoot traits of maize seedlings under cold stress. Theoret. Appl. Genetics. 109:618-629.

Joosen, R.V.L., Kodde, J., Willems, L.A.J., Ligterink, W., van der Plas, L.H.W., Hilhorst, H.W.M. 2010. Germinator: a software package for high-throughput scoring and curve fitting of Arabidopsis seed germination. Plant J. 62:148-159.

Joshi, R., Ramanarao, M.V., Baisakh, N. 2013. Arabidopsis plants constitutively overexpressing a myo-inositol 1-phosphate synthase gene (SaINO1) from the halophyte smooth cordgrass exhibits enhanced level of tolerance to salt stress. Plant Physiol. Biochem. 65:61-66.

Kaymak, H.C. 2012. The relationships between seed fatty acids profile and seed germination in cucurbit species. Zemdirbyste. 99:299-304.

Klages, K., Boldingh, H., Smith, G.S. 1999. Accumulation of myo-inositol in actinidia seedlings subjected to salt stress. Ann. Bot. 84:521-527.

Mansour, M.M.F. 2000. Nitrogen containing compounds and adaptation of plants to salinity stress. Biol. Plant. 43:491-500.

Meis, S.J., Fehr, W.R., Schnebly, S.R. 2003. Seed source effect on field emergence of soybean line with reduced phytate and raffinose saccharides. Crop Sci. 43:1336-1339.

Michell, R.H. Evolution of the diverse biological roles of inositols. In: Biochem. Soc. Symp. 2007.

Nasri, N., Kaddour, R., Rabhi, M., Plassard, C., Lachaal, M. 2011. Effect of salinity on germination, phytase activity and phytate content in lettuce seedling. Acta Physiol. Plant. 33:935-942.

Piwowarczyk, B., Tokarz, K., Kamińska, I. 2016. Responses of grass pea seedlings to salinity stress in in vitro culture conditions. Plant Cell. Tiss. Org. 124:227-240.

Raboy, V. 2009. Approaches and challenges to engineering seed phytate and total phosphorus. Plant Sci. 177:281-296.

Raychaudhuri, A., Majumder, A.L. 1996. Salinity-induced enhancement of L-myo-inositol 1-phosphate synthase in rice (Oryza sativa L.). Plant Cell Environ. 19:1437-1442.

Rose, T.J., Liu, L., Wissuwa, M. 2013. Improving phosphorus efficiency in cereal crops: Is breeding for reduced grain phosphorus concentration part of the solution? Front. Plant Sci. 4:444.

Saxena, S.C., Salvi, P., Kaur, H., Verma, P., Petla, B.P., Rao, V., Kamble, N., Majee, M. 2013. Differentially expressed myo-inositol monophosphatase gene (CaIMP) in chickpea (Cicer arietinum L.) encodes a lithium- 
sensitive phosphatase enzyme with broad substrate specificity and improves seed germination and seedling growth under abiotic stresses. J. Exp. Bot. 64:5623-5639.

Schneider, S., Schneidereit, A., Kai, R.K., Hajirezaei, M.R., Gramann, M., Hedrich, R., Sauer, N. 2006. Arabidopsis inositol transporter4 mediates high-affinity $\mathrm{H}^{+}$symport of myoinositol across the plasma membrane. Plant Physiol. 141:565.

Suzuki, M., Tanaka, K., Kuwano, M., Yoshida, K.T. 2007. Expression pattern of inositol phosphate-related enzymes in rice (Oryza sativa L.): Implications for the phytic acid biosynthetic pathway. Gene 405:55-64.

Talbi Zribi, O., Abdelly, C., Debez, A. 2011. Interactive effects of salinity and phosphorus availability on growth, water relations, nutritional status and photosynthetic activity of barley (Hordeum vulgare L.). Plant Biol. 13:872-880.

Tan, L., Chen, S., Wang, T., Dai, S. 2013. Proteomic insights into seed germination in response to environmental factors. Proteomics 13:1850-1870.

Voigt, E.L., Almeida, T.D., Chagas, R.M., Ponte, L.F.A., Viégas, R.A., Silveira, J.A.G. 2009. Source-sink regulation of cotyledonary reserve mobilization during cashew (Anacardium occidentale) seedling establishment under $\mathrm{NaCl}$ salinity. J. Plant Physiol. 166:80-89.

Wang, F., Rose, T., Jeong, K., Kretzschmar, T., Wissuwa, M. 2016. The knowns and unknowns of phosphorus loading into grains, and implications for phosphorus efficiency in cropping systems. J. Exp. Bot. 67:12211229.

White, P.J., Veneklaas, E.J. 2012. Nature and nurture: the importance of seed phosphorus content. Plant Soil 357:1-8.

Witzel, K., Weidner, A., Surabhi, G.K., Varshney, R.K., Kunze, G., Buck-Sorlin, G.H., Börner, A., Mock, H.P. 2010. Comparative analysis of the grain proteome fraction in barley genotypes with contrasting salinity tolerance during germination. Plant Cell Environ. 33:211-222.

Xu, E., Chen, M., He, H., Zhan, C., Cheng, Y., Zhang, H., Wang, Z. 2016. Proteomic analysis reveals proteins involved in seed imbibition under salt stress in rice. Front. Plant Sci. 7

Xue, W., Yan, J., Zhao, G., Jiang, Y., Cheng, J., Cattivelli, L., Tondelli, A. 2017. A major QTL on chromosome 7HS controls the response of barley seedling to salt stress in the Nure $\times$ Tremois population. BMC Genet. 18:79.

Xue, W.T., Gianinetti, A., Wang, R., Zhan, Z.J., Yan, J., Jiang, Y., Fahima, T., Zhao, G., Cheng, J.P. 2016. Characterizing barley seed macro- and micro-nutrients under multiple environmental conditions. Cereal Res. Commun. 44:639-649.

Zhang, H., Irving, L.J., McGill, C., Matthew, C., Zhou, D., Kemp, P. 2010. The effects of salinity and osmotic stress on barley germination rate: sodium as an osmotic regulator. Ann. Bot. 106:1027-1035.

Zhang, M., Nyborg, M., McGill, W.B. 1990. Phosphorus concentration in barley (Hordeum vulgare L.) seed: Influence on seedling growth and dry matter production. Plant Soil 122:79-83.

Zhou, S., Sekizaki, H., Yang, Z.H., Sawa, S., Pan, J. 2010. Phenolics in the seed coat of wild soybean (Glycine soja) and their significance for seed hardness and seed germination. J. Agr. Food Chem. 58:10972-10978.

Zhu, M., Zhou, M., Shabala, L., Shabala, S. 2015. Linking osmotic adjustment and stomatal characteristics with salinity stress tolerance in contrasting barley accessions. Funct. Plant Biol. 42:252-263.

\section{Electronic Supplementary Material (ESM)}

Electronic Supplementary Material (ESM) associated with this article can be found at the website of CRC at https://akademiai.com/loi/0806

Electronic Supplementary Table S1. pH, organic matter content and element concentrations in the soil of three field locations in China

Electronic Supplementary Table S2. Two-way ANOVA of seed components from four environments 
Electronic Supplementary Table S3. Two-way ANOVA of either root length (RL) or shoot length (SL) from four environments

Electronic Supplementary Figure S1. Comparison of averages of barley seed components among four environments. Bars represent mean \pm SD. Different lowercase letters indicate significant differences at $p<0.05$ (Tukey HSD). Sta: starch, TSP: total soluble protein, Phy: phytate, Phe: total phenolics, Fla: total flavonoids, $\mathrm{P}_{\mathrm{i}}$ : inorganic phosphorus

Electronic Supplementary Figure S2. Correlations between seed components and seedling traits. Correlations were conducted with dataset from four environments. Solid and dotted lines refer to positive and negative correlations, respectively. Thin and wider lines indicate coefficients $r<0.4$ and $r>0.4$ respectively. Sta: starch, TSP: total soluble protein, Phy: phytate, Phe: total phenolics, Fla: total flavonoids, $\mathrm{P}_{\mathrm{i}}$ : inorganic phosphorus 